\title{
RESULTS ON PREPROCESSING, POSTPROCESSING AND NODE PROCESSING IN INDUSTRIAL LP AND MIP MODELS
}

\section{Van hamme, Beyers Innovative Software Brasschaat, Belgium A. Van Looveren, Beyers Innovative Software Brasschaat, Belgium}

OMP, a general solver for industrial LP and MIP models, is based on the simplex and branch and bound algorithms. Performance has been substantially improved through the implementation of various techniques, which reduce the model size and prune the branch and bound tree. Furthermore, the basic algorithms have been made faster, and the choice of branching variables has been sophisticated.

The model reduction techniques include variable and constraint elimination and substitution. Pruning of the branch and bound tree is obtained by sharpening variable bounds, applying coefficient reduction to the constraints, and generating cuts.

An overview of the various algorithms is presented. The modifications have been tested on a set of practical industrial LP and MIP models. Test results are evaluated on the basis of solution time. Improvements in LP models range from a factor 2 to 10. For MIP models, even more dramatic reductions in solution time have been obtained. Problems which could not $b_{i}$; solved within a reasonable amount of ime, are now solved with proven optimality within minutes. 